\section{Chemotaxis and the cell surface-area problem}

\section{Maurice B. Hallett ${ }^{1}$, Christopher J. von Ruhland ${ }^{2}$ and Sharon Dewitt ${ }^{1}$}

The review by Robert Kay and colleagues (Changing directions in the study of chemotaxis. Nature Rev. Mol. Cell Biol. 9, $455-463(2008))^{1}$ is a masterly and muchneeded overview of the problems that prevent a full understanding of the underlying mechanisms behind directional finding and chemotaxis by Dictyostelium discoideum and neutrophils. Kay and colleagues discussed the neglected but important topic of the 'surface-area problem' - the mechanism by which the apparent surface area of the chemotactic cell expands (and contracts). It has been argued that without the ability to expand its surface membrane area, cell

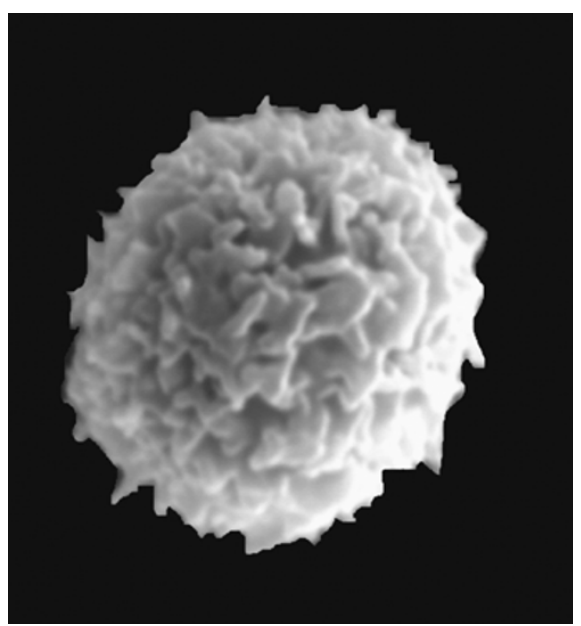

Figure 1 | Scanning electron micrograph of a human neutrophil, which shows its extensively wrinkled surface. The wrinkles could provide the solution to the "surface-area problem' by acting as a reservoir of extra membrane and by permitting changes in cell surface area as the cell undergoes chemotaxis and phagocytosis. polarization, pseudopod formation, phagocytosis and chemotaxis would not be possible, and thus actin polymerization and other cytoplasmic changes are subordinate to membrane expansion ${ }^{2,3}$. However, Kay and colleagues have underestimated the surfacearea problem for neutrophils that increase their surface by far more than the $20-30 \%$ increase in surface area reported during D. discoideum migration ${ }^{4}$.

Neutrophils that undergo phagocytosis ${ }^{2}$ or undergo a transition from a spherical to a flattened morphology ${ }^{3}$ can approximately double their apparent surface area. In their review, Kay and colleagues suggest that 'folds' in the cell surface as possible reservoirs of additional membrane are unlikely, and focus on endocytic cycling as the potential mechanism. However, scanning electron microscopy of neutrophils show that this cell type has a wrinkled surface ${ }^{5}$, which we estimate could double the apparent cell surface area (FIG. 1). Furthermore, the wrinkles disappear during expansion of the apparent surface area by osmotic swelling, and quantification shows that this membrane reservoir produces an additional surface-area increase of approximately $100 \%$ (REF. 6). The unwrinkling of the membrane can also be achieved by pulling the neutrophil membrane by an antibody-coated bead $^{7}$ or by suction through a micropipette ${ }^{8,9}$, both producing extra membrane (and thus increasing the surface area). Mathematical modelling of the kinetics and forces that are required suggests that this extra membrane results from the unfurling of plasma-membrane wrinkles, which are held in place by a 'molecular velcro' (REF. 10). Significantly, the force required to 'unwrinkle' the membrane is significantly reduced during phagocytosis ${ }^{10}$, which suggests that the velcro holding the wrinkles together can be released by intracellular signals that are associated with phagocytotic stimulation. We have suggested that these signals might include the cleavage of proteins that hold the wrinkles in place ${ }^{2,3}$, and involve $\mathrm{Ca}^{2+}$ activation of $\mu$-calpain ${ }^{11}$. Thus, although endocytic cycling has been suggested as a way of replacing integrin to the front of neutrophils ${ }^{12}$, the wrinkled cell surface must not be discounted as a possible solution to the surface-area problem as, in neutrophils, it is a potentially very large reservoir for providing apparent membrane expansion.

'Neutrophil Signalling Group, School of Medicine, Cardiff University, Cardiff CF14 4XN, UK.

${ }^{2}$ Medical Microscopy Sciences, School of Medicine, Cardiff University, Cardiff CF14 4XN, UK

Correspondence to M.B.H. e-mail:hallettmb@cardiff.ac.uk doi:10.1038/nrm2419-c1

Kay, R. R., Landridge, P., Traynor, D. \& Hoeller, O. Changing directions in the study of chemotaxis. Nature Rev. Mol. Cell Biol. 9, 455-463 (2008). 2. Hallett, M. B. \& Dewitt, S. Ironing out the wrinkles of neutrophil phagocytosis: membrane reservoirs for surface area expansion. Trends Cell Biol. 17, 209-214 (2007)

3. Dewitt, S. \& Hallett, M. B. Leukocyte membrane "expansion": A central mechanism for leukocyte extravasation. J. Leukoc. Biol. 81, 1160-1164 (2007).

4. Traynor, D. \& Kay, R. R. Possible roles of the endocytic cycle in cell motility. J. Cell Sci. 120, 2318-2327 (2007).

5. Bessis, M. Living Blood Cells and their Ultrastructure (Springer, Berlin, 1973).

6. Ting-Beall, H. P., Needham, D. \& Hochmuth, R. M. Volume and osmotic properties of human neutrophils. Blood 81, 2774-2780 (1993).

7. Shao, J. Y. \& Hochmuth, R. M. Micropipette suction for measuring piconewton forces of adhesion and tether formation. Biophys. J. 71, 2892-2901 (1996)

8. Evans. E., Leung, A. \& Zhelev, D. Synchrony of cell spreading and contraction force as phagocytes engulf large pathogens. J. Cell Biol. 122, 1295-1300 (1993).

9. Herant, M., Heinrich, V. \& Dembo, M. Mechanics of neutrophil phagocytosis: experiments and quantitative models. J. Cell Sci. 119, 1903-1913 (2006).

10. Herant, M., Heinrich. V. \& Dembo, M. Mechanics of neutrophil phagocytosis: behavior of the cortical tension. J. Cell Sci. 118, 1789-1797 (2005).

11. Dewitt, S. \& Hallett, M. B. Cytosolic free $\mathrm{Ca}^{2+}$ changes and calpain activation are required for $\beta 2$ integrinaccelerated phagocytosis by human neutrophils. J. Cell Biol. 159, 181-189 (2002).

12. Lawson, M. A. \& Maxfield, F. R. $\mathrm{Ca}^{2+}$-and calcineurindependent recycling of an integrin to the front of migrating neutrophils. Nature 377, 75-79 (1995) 